2017 , volume 6, issue 2

Eden, D., Huffman, P., \& Holman, J. (2017). Heavy-tailed distributions and the Canadian stock market returns. Copernican Journal of Finance \& Accounting, 6(2), 9-21. http://dx.doi.org/10.12775/ CJFA.2017.007

\author{
David Eden* \\ Bank of Canada \\ Paul Huffman ${ }^{* *}$ \\ University of Manitoba \\ John Holman ${ }^{* * *}$ \\ Illinois State University
}

\title{
HEAVY-TAILED DISTRIBUTIONS \\ AND THE CANADIAN STOCK MARKET RETURNS
}

Keywords: Value at Risk, GSPTSE, Skewed $t$ distribution.

\section{J E L Classification: C46, C58, G10.}

Abstract: Many of financial engineering theories are based on so-called "complete markets" and on the use of the Black-Scholes formula. The formula relies on the assumption that asset prices follow a log-normal distribution, or in other words, the daily fluctuations in prices viewed as percentage changes follow a Gaussian distribution. On the contrary, studies of actual asset prices show that they do not follow a log-normal distribution. In this paper, we investigate several widely-used heavy-tailed distributions. Our results indicate that the Skewed $t$ distribution has the best empirical performance

Date of submission: August 29, 2017; date of acceptance: October 23, 2017.

* Contact information: deden0817@gmail.com, Financial Markets Department, Bank of Canada, 234 Wellington Street, Ottawa, Ontario, Canada, phone: 16137835643.

** Contact information: Department of Economics, University of Manitoba, Winnipeg, Manitoba, Canada.

${ }^{* * *}$ Contact information: Department of Economics, Illinois State University, Normal, IL, United States. 
in fitting the Canadian stock market returns. We claim the results are valuable for market participants and the financial industry.

\section{IIINTRODUCTION}

According to IMF (2014), Canada has a well-developed financial system, with assets totalling about 500 percent of GDP, and dominated by a variety of industry participants. The equities market is mature, with a total market capitalization of nearly Canadian $\$ 2.1$ trillion (115 percent of GDP) at end of 2012. Toronto Stock Exchange (TSX) is one of the world's largest stock exchanges. It is the ninth largest exchange in the world by market capitalization. The S\&P/TSX Composite Index (GSPTSE) is the benchmark Canadian index, representing roughly $70 \%$ of the total market capitalization on the TSX with about $250 \mathrm{com}$ panies included in it. The TSX is made up of over 1,500 companies in total.

Guo (2017a) introduced several different types of heavy-tailed distribution and show the Skewed $t$ distribution generates the best goodness of fit and risk measurements. Following Guo (2017a), we are also interested in the empirical performance of various types of heavy-tailed distributions in fitting the Canadian stock market returns. Lots of financial models are based on the assumption that asset prices follow a log-normal distribution, or in other words, the daily fluctuations in prices viewed as percentage changes follow a normal distribution. Therefore, our results have rich implications for the financial models and the financial industry in Canada, as these models have been widely used in practices.

\section{RESEARCH METHODOLOGY AND THE COURSE OF THE RESEARCH PROCESS}

In probability theory, heavy-tailed distributions are probability distributions whose tails are not exponentially bounded, and they have heavier tails than the exponential distribution as defined in Asmussen (2003). In finance, as Cont (2001) pointed out, it a stylized fact that the distribution of asset returns display a power-law or Pareto-like tail, with a tail index which is finite, higher than two and less than five for most data sets studied. In particular this excludes stable laws with infinite variance and the normal distribution. However, Cont also mentioned that the precise form of the tails is difficult to determine. A recent survey can also be found in Ibragimov (2009). 
Since precision form of the tails is the key for financial risk management, there are increasing interests in development of heavy-tailed distributions for asset returns in the recent decades. For instance, Glasserman, et al. (2002) developed multivariate $t$ distribution for computing portfolio value-at-risk (VAR) when underlying risk factors follow heavy-tailed distributions. Bradley and Taqqu (2003) reviewed recent development on how the heavy-tailed distributions affect several aspects of financial portfolio theory and risk management. Using foreign exchange rate returns as an example, Mittnik and Paolella (2003) demonstrated that GARCH models with stable Paretian innovations perform better in predicting the downside risk of financial assets that use of normal or Student's $t$ GARCH models. Ahn, Kim and Ramaswami (2012) studied the class of log phase-type (LogPH) distributions as a parametric alternative in fitting heavy tailed data, and demonstrated its superior performance in insurance risk management. Guo (2017a) investigated five widely-used statistical distributions in fitting SP 500 index returns: normal, Student's $t$, Skewed $t$, normal inverse Gaussian (NIG), and generalized hyperbolic (GH) distributions. Guo demonstrated the Skewed $t$ distribution introduced in Hansen (1994) has the best goodness of fit and generates suitable stress test scenarios.

There are some researchers investigating the Canadian stock market in a multivariate framework. For instance, Theodossiou and Lee (1993) provides additional insight into the nature and degree of interdependence of stock markets of the United States, Japan, the United Kingdom, Canada, and Germany, and it reports the extent to which volatility in these markets influences expected returns. The analysis uses the multivariate GARCH-M model. Although they are considered weak, statistically significant mean spillovers radiate from stock markets of the U.S. to the U.K., Canada, and Germany, and then from the stock markets of Japan to Germany. No relation is found between conditional market volatility and expected returns. Strong time-varying conditional volatility exists in the return series of all markets. The own-volatility spillovers in the U.K. and Canadian markets are insignificant, supporting the view that conditional volatility of returns in these markets is "imported" from abroad, specifically from the U.S. Significant volatility spillovers radiate from the U.S. stock market to all four stock markets, from the U.K. stock market to the Canadian stock market, and from the German stock market to the Japanese stock market. Their results are robust and no changes occur in the correlation structure of returns over time. Karolyi (2012) examines the short-run dynamics of returns and volatility for stocks traded on the New York and Toronto stock exchanges. 
The main finding is that inferences about the magnitude and persistence of return innovations that originate in either market and that transmit to the other market depend importantly on how the cross-market dynamics in volatility are modeled. Moreover, much weaker cross-market dynamics in returns and volatility prevail during later subperiods and especially for Canadian stocks with shares dually listed in New York. Some other research could be found in Park and Switzer (1995), Ramchand and Susmel (1998), Sadorsky (1999), and so on.

In this paper, we follow Guo's approach, but focus on the Canadian stock market returns. The remainder of the paper is organized as follows. Firstly, we introduce the heavy-tailed distributions in Guo (2017a). Secondly, we discuss the data. Then, the estimation results are presented. Finally, we conclude.

\section{THE HEAVY-TAILED DISTRIBUTIONS}

Following Guo (2017a), we are interested the four following types of heavytailed distribution in addition to the normal distribution: (i) the Student's $t$ distribution; (ii) the Skewed $t$ distribution; (iii) the normal inverse Gaussian distribution (NIG); and (iv) the generalized hyperbolic distribution (GH). All the distributions have been standardized to ensure mean and standard deviation equal to zero and one respectively. Their probability density functions are given as follows.

(i) Student's $t$ distribution:

$$
f\left(e_{t} \mid v\right)=\frac{\Gamma\left(\frac{v+1}{2}\right)}{\Gamma\left(\frac{v}{2}\right)[(v-2) \pi]^{1 / 2}}\left(1+\frac{e_{t}^{2}}{(v-2)}\right)^{-\frac{v+1}{2}},
$$

where $v$ indicates degrees of freedom and $e_{t}$ is daily equity market index return.

(ii) Skewed $t$ distribution:

$$
f\left(e_{t} \mid v, \beta\right)=\left\{\begin{array}{ll}
b c\left(1+\frac{1}{v-2}\left(\frac{b e_{t}+a}{1-\beta}\right)^{2}\right)^{-(v+1) / 2} & e_{t}<-a / b \\
b c\left(1+\frac{1}{v-2}\left(\frac{b e_{t}+a}{1+\beta}\right)^{2}\right)^{-(v+1) / 2} & e_{t} \geq-a / b
\end{array},\right.
$$


where $e_{t}$ is the standardized $\log$ return, and the constants $a, b$ and $c$ are given by

$$
a=4 \beta c\left(\frac{v-2}{v-1}\right), b^{2}=1+3 \beta^{2}-a^{2}, \text { and } c=\frac{\Gamma\left(\frac{v+1}{2}\right)}{\sqrt{\pi(v-2)} \Gamma\left(\frac{v}{2}\right)} .
$$

The density function has a mode of $-a / b$, a mean of zero, and a unit variance. The density function is skewed to the right when $\beta>0$, and vice-versa when $\beta<0$. The Skewed $t$ distribution specializes to the standard Student's $t$ distribution by setting the parameter $\beta=0$.

(iii) Normal inverse Gaussian distribution (NIG):

$$
f\left(e_{t} \mid \mu, \alpha, \beta, \delta\right)=\frac{\alpha \delta K_{1}\left(\alpha \sqrt{\delta^{2}+\left(e_{t}-\mu\right)^{2}}\right)}{\pi \sqrt{\delta^{2}+\left(e_{t}-\mu\right)^{2}}} \exp \left(\delta \sqrt{\alpha^{2}-\beta^{2}}+\beta\left(e_{t}-\mu\right)\right)
$$

where $K_{\lambda}(\cdot)$ (is the modified Bessel function of the third kind and index $\lambda=0$ and $\alpha>0$. The NIG distribution is specified as in Prause (1999). The NIG distribution is normalized by

setting $\mu=-\frac{\delta \beta}{\sqrt{\alpha^{2}-\beta^{2}}}$ and $\delta=\frac{\left(\sqrt{\alpha^{2}-\beta^{2}}\right)^{3}}{\alpha^{2}}$,

which implies $E\left(e_{1}\right)=0$ and $\operatorname{Var}\left(e_{t}\right)=1$.

(iv) Generalized hyperbolic distribution:

$$
f\left(e_{t} \mid p, b, g\right)=\frac{g^{p}}{\sqrt{2 \pi}\left(b^{2}+g^{2}\right)^{\frac{1}{2}\left(p-\frac{1}{2}\right)} d(p, b, g) K_{p}(g)} q\left(\frac{e_{t}-m(p, b, g)}{d(p, b, g)} ; p, b, g\right),
$$

where $\tilde{R}_{n} \triangleq \frac{K_{n+p}(g)}{g^{n} K_{p}(g)}, d(p, b, g) \triangleq\left[\tilde{R}_{1}+b^{2}\left\{\tilde{R}_{2}-\tilde{R}_{1}^{2}\right\}\right]^{-\frac{1}{2}} \geq 0$,

and $m(p, b, g) \triangleq-b d(p, b, g) \tilde{R}_{1} \cdot p, b$ are $g$ parameters. The generalized hyperbolic distribution is a standardized version of Prause (1999). 


\section{DATA}

We fit the heavy tailed distributions with the normalized equity market index returns of Canada. We choose the GSPTSE index as it is the benchmark Canadian index, representing roughly $70 \%$ of the total market capitalization on the Toronto Stock Exchange with about 250 companies included in it. We collected the standardized GSPTSE daily dividend-adjusted close returns from Yahoo Finance for the period from July 2, 1979 to July 14, 2017, covering all the available data in Yahoo Finance. There are in total 9682 observations. Figure 1 illustrates the dynamics of GSPTSE returns. The figure exhibits significant volatility clustering, and the two biggest groups of spikes were observed during the periods of the Black Monday Stock Crash and the recent financial crisis.

Figure 1. GSPTSE Returns

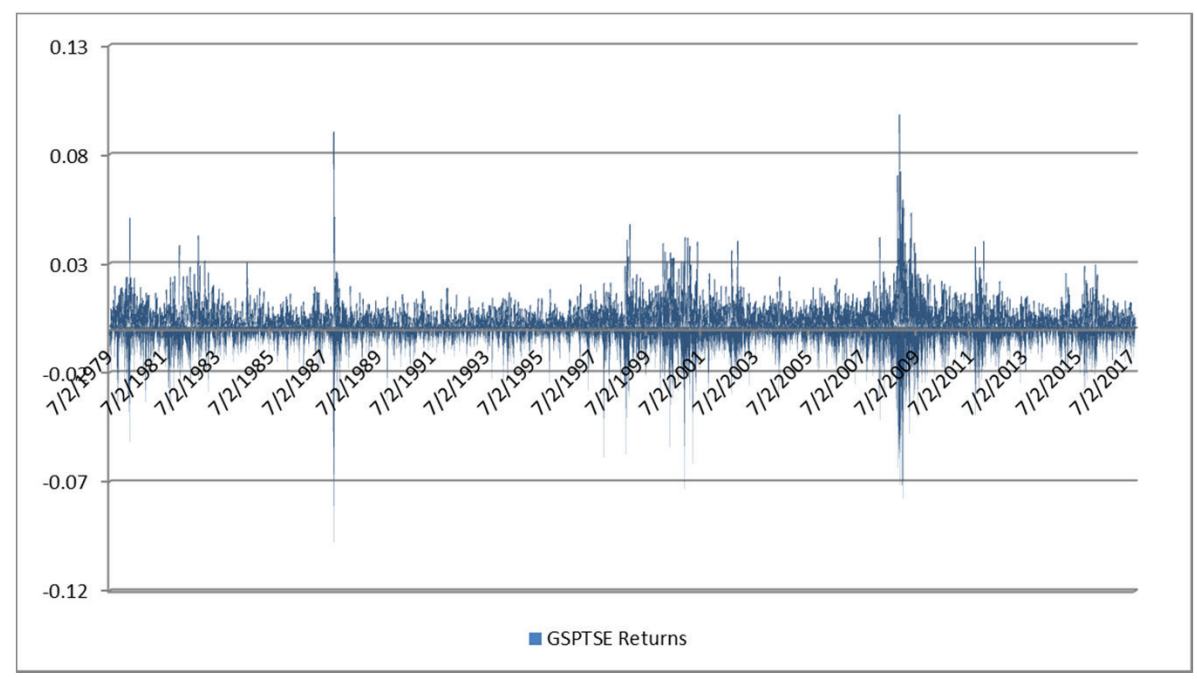

S o u r c e : authors' analysis.

Table 1 exhibits basic statistics of the GSPTSE returns. The results show the GSPTSE daily returns are leptokurtotic and negatively skewed. The extreme downside move is slightly less than the extreme upside move, which is at odds with most of major stock market indexes over the world. 
Table 1. Descriptive statistics

\begin{tabular}{|c|c|c|c|c|c|}
\hline \hline $\min$ & $\max$ & $\operatorname{mean}$ & std & skewness & kurtosis \\
\hline \hline$-11.13 \%$ & $9.82 \%$ & $0.03 \%$ & $0.96 \%$ & -0.64 & 12.35 \\
\hline \hline
\end{tabular}

S o u r c e : authors' analysis.

Figure 2 is the histogram of the raw data. We fit the returns by the Gaussian distribution, and the figure clearly rejects the null hypothesis of the Gaussian distribution. Also, figure 2 confirms the negative skewness and heavy kurtosis as shown in table 1.

Figure 2. GSPTSE returns

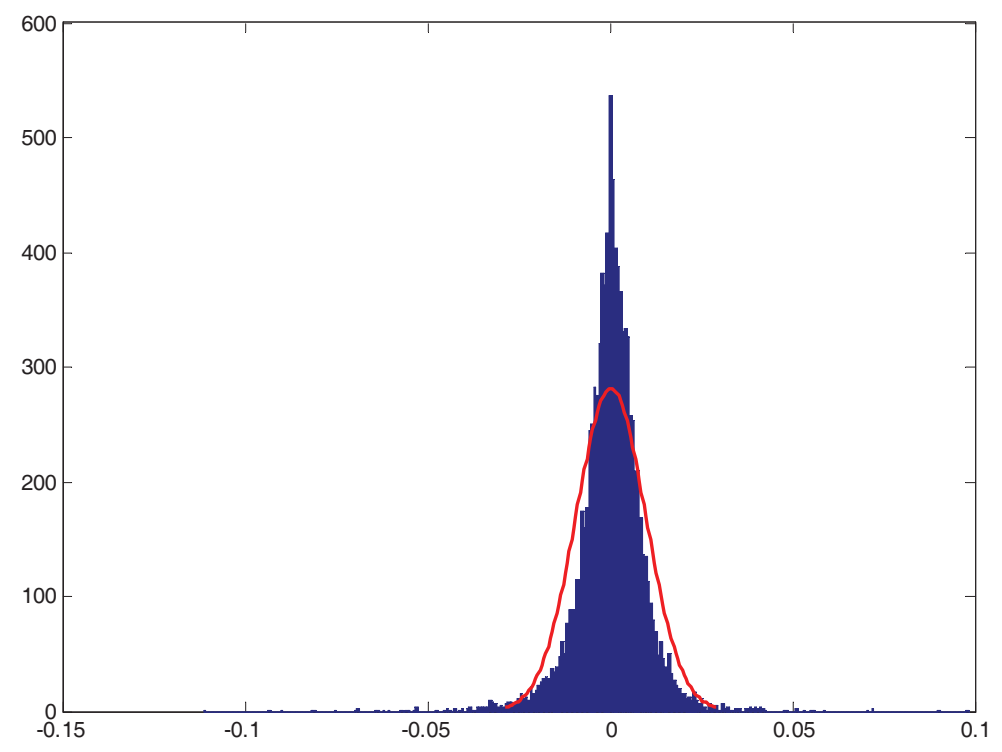

S o u r c e : authors' analysis. 


\section{EMPIRICAL RESULTS}

\section{Parameters Estimation}

The raw return series is fitted by the maximum likelihood estimation (MLE) method and the estimation results of the key parameters are given in table 2 . The series is normalized to allow zero mean and unit standard deviation. All the parameters are significantly different from zero at 10\% significance level.

Table 2. Estimated values of key parameters

\begin{tabular}{|l|l|l|l|l|l|}
\hline \hline & \multicolumn{1}{|c|}{ Normal } & \multicolumn{1}{|c|}{ Student's $t$} & \multicolumn{1}{|c|}{ Skewed $t$} & \multicolumn{1}{|c|}{ NIG } & \multicolumn{1}{c|}{$\begin{array}{c}\text { Generalized } \\
\text { Hyperbolic }\end{array}$} \\
\hline \hline Symmetric & $\mathrm{Y}$ & $\mathrm{Y}$ & $\mathrm{N}$ & $\mathrm{N}$ & $\mathrm{N}$ \\
\hline Fat-tailed & $\mathrm{N}$ & $\mathrm{Y}$ & $\mathrm{Y}$ & $\mathrm{Y}$ & $\mathrm{Y}$ \\
\hline $\begin{array}{l}\text { Estimated } \\
\text { Parameters }\end{array}$ & & $\mathrm{Nu}=4.72$ & $\begin{array}{l}\text { Nu=4.73; } \\
\text { beta }=-0.027\end{array}$ & $\begin{array}{l}\text { alpha }=1.35 ; \\
\text { beta }=-0.032\end{array}$ & $\begin{array}{l}\mathrm{p}=-1.323 ; \\
\mathrm{b}=-.042 ; \\
\mathrm{g}=0.127\end{array}$ \\
\hline \hline
\end{tabular}

S o u r c e : authors' analysis.

\section{Goodness of Fit}

Following Huber-Carol, et al. (2002) and Taeger and Kuhnt (2014), we compare the four heavy-tailed distributions and the benchmark normal distribution in fitting the GSPTSE daily returns through four different criteria: (i) Kolmogorov-Smirnov statistic; (ii) Cramer-von Mises criterion; (iii) Anderson-Darling test; and (iv) Akaike information criterion (AIC).

(i) Kolmogorov-Smirnov statistic is defined as the maximum deviation between empirical CDF (cumulative distribution function) $F_{n}(x)$ and tested $\operatorname{CDF} F(x)$ :

$$
D_{n}=\sup _{x}\left|F_{n}(x)-F(x)\right|,
$$

where $F_{n}(x)=\frac{1}{n} \sum_{i=1}^{n} I_{[-\infty, x]}\left(X_{i}\right)$. 
(b) Cramer-von Mises criterion is defined as the average squared deviation between empirical CDF and tested CDF:

$$
T=n \int_{-\infty}^{\infty}\left[F_{n}(x)-F(x)\right]^{2} d F(x)=\frac{1}{12 n}+\sum_{i=1}^{n}\left[\frac{2 i-1}{2 n}-F_{n}\left(x_{i}\right)\right]^{2},
$$

(c) Anderson-Darling test is defined as the weighted-average squared deviation between empirical CDF and tested CDF:

$$
A=n \int_{-\infty}^{\infty} \frac{\left(F_{n}(x)-F(x)\right)^{2}}{F(x)(1-F(x))} d F(x),
$$

and the formula for the test statistic to assess if data comes from a tested distribution is given by:

$$
A^{2}=-n-\sum_{i=1}^{n} \frac{2 i-1}{n}\left[\ln \left(F\left(x_{i}\right)\right)+\ln \left(1-F\left(x_{i}\right)\right)\right] .
$$

(d) Akaike information criterion (AIC) is defined as:

$$
A I C=-2 k-2 \ln (L),
$$

where $L$ is the maximum value of the likelihood function for the model, and $k$ is the number of estimated parameters in the model.

The comparison results are showed in table 3 , indicating the Skewed $t$ distribution has the best goodness of fit compared with other selected types of distribution, followed by the generalized hyperbolic distribution, and the Student's $t$ distribution.

Table 3. Comparison of selected types of distribution

\begin{tabular}{|l|c|c|c|c|c|}
\hline \hline & Normal & Student's $t$ & Skewed $t$ & NIG & Generalized Hyperbolic \\
\hline \hline K-S Test & 0.039 & 0.030 & 0.028 & 0.031 & 0.029 \\
\hline Cv-M Test & 0.056 & 0.048 & 0.044 & 0.049 & 0.047 \\
\hline A-D Test & 1.97 & 1.62 & 1.49 & 1.58 & 1.52 \\
\hline AIC & 32555 & 31158 & 30643 & 31431 & 30946 \\
\hline \hline
\end{tabular}

S o u r c e : authors' analysis. 


\section{Stress Test Scenarios}

To analyze how these distributions perform in financial risk management, we take advantage of the concept of Value at Risk (VaR) to calculate stress test scenarios. Here, VaR is defined as: for a given position, time horizon, and probability $p$, the $p$ VaR is defined as a threshold loss value, such that the probability that the loss on the position over the given time horizon exceeds this value is $p$. With the estimated parameters in Section 4.1, we calculate VaRs for different confidence levels:

$$
\operatorname{VaR}_{\alpha}\left(e_{t}\right)=\inf \left\{e \in \mathbb{R}: P\left(e_{t}>e\right) \leq 1-\alpha\right\},
$$

where $\alpha \in(0,1)$ is the confidence level. We select the following levels for downside moves: $\{99.99 \%, 99.95 \%, 99.9 \%, 99.5 \%, 99 \%\}$, and for upside moves: $\{0.01 \%, 0.05 \%, 0.1 \%, 0.5 \%, 1 \%\}$. From Equation (9), the stress test scenarios based on the VaR levels are given as in table 4. Table 4 indicates that the Skewed $t$ distribution has the closest VaRs to the nonparametric historical VaRs compared with other types of distributions.

Table 4. Scenarios for GSPTSE shocks

\begin{tabular}{|l|c|c|c|c|c|}
\hline \hline \multicolumn{7}{|c|}{ Left Tail } \\
\hline \hline Confidence & $99.99 \%$ & $99.95 \%$ & $99.90 \%$ & $99.50 \%$ & $99.00 \%$ \\
\hline Empirical & $-12.17 \%$ & $-11.00 \%$ & $-8.47 \%$ & $-6.25 \%$ & $-4.92 \%$ \\
\hline Normal & $-6.52 \%$ & $-5.65 \%$ & $-5.20 \%$ & $-4.47 \%$ & $-4.02 \%$ \\
\hline T & $-11.60 \%$ & $-10.25 \%$ & $-9.25 \%$ & $-7.32 \%$ & $-5.97 \%$ \\
\hline Skewed T & $-12.02 \%$ & $-11.07 \%$ & $-8.10 \%$ & $-6.40 \%$ & $-5.15 \%$ \\
\hline NIG & $-10.15 \%$ & $-9.30 \%$ & $-7.92 \%$ & $-7.15 \%$ & $-5.90 \%$ \\
\hline GH & $-11.65 \%$ & $-10.45 \%$ & $-8.77 \%$ & $-6.80 \%$ & $-5.40 \%$ \\
\hline \hline & & & & & \\
\hline \hline Confidence & $0.01 \%$ & $0.05 \%$ & $0.10 \%$ & $0.50 \%$ & $1.00 \%$ \\
\hline Empirical & $10.10 \%$ & $7.85 \%$ & $7.05 \%$ & $5.35 \%$ & $4.35 \%$ \\
\hline Normal & $6.52 \%$ & $5.65 \%$ & $5.20 \%$ & $4.47 \%$ & $4.02 \%$ \\
\hline T & $11.60 \%$ & $10.25 \%$ & $9.25 \%$ & $7.32 \%$ & $5.97 \%$ \\
\hline \hline
\end{tabular}


Table 4. Scenarios for GSPTSE shocks

\begin{tabular}{|l|c|c|c|c|c|}
\hline \hline \multicolumn{7}{|c|}{ Right Tail } \\
\hline \hline NIG & $11.17 \%$ & $9.85 \%$ & $8.17 \%$ & $7.00 \%$ & $5.90 \%$ \\
\hline GH & $12.05 \%$ & $10.07 \%$ & $8.47 \%$ & $6.90 \%$ & $5.27 \%$ \\
\hline \hline
\end{tabular}

S o u r c e : authors' analysis.

\section{CONCLUSIONS}

Financial market participants are always interested in the tail parts of the statistical distributions, since precision of the tails are crucial for financial risk management. Thus, statistical distributions which can capture the tail parts of the empirical distribution would be particular interesting for the market participants. In this paper, we focus on the GSPTSE Index, the most important risk factor in the equity market in Canada, and develop a methodology to construct its stress test scenarios. By comparing empirical performance of different statistical distributions, our results show the Skewed $t$ distribution could generate the most suitable risk management scenarios for GSPTSE Index.

There are two directions for further research. First, one may jointly consider the risk factor of stock market returns with other risk factors, such as unemployment and interest rates, and investigate how to extend the Skewed $t$ distribution to the tail-dependence framework. Second, one may introduce the heavy-tailed distributions to the generalized autoregressive conditional heteroskedasticity (GARCH) framework as in Guo (2017b, 2017c) and study their implications in financial risk management, and the extension would be similar as the studies in Day and Diamond (2017), Maree (2017) and Maree, Carr and Howard (2017). Finally, one might consider a portfolio with multiple assets and consider risk management of heavy tails across assets in a multivariate GARCH framework as in Karolyi (2012) and Guo (2017d). As in Guo (2017d), to capture the tail risks across assets, one has to rely on the copula approach. However, the Skewed $t$ distribution with copula still project better risk measures, at least for the case of two assets. As pointed out in Cont (2001), there are several other stylized facts observed in the financial markets: such as the correlation of volume and volatility. It is interesting to investigate if the heavy tails are also observed in the volume series and also if the Skewed $t$ distribution has a similar superior performance in fitting the volume growth. All these are left for future research. 


\section{REFERENCES}

Ahn, S., Kim, Joseph H.T., \& Ramaswami. V. (2012). A new class of models for heavy tailed distributions in finance and insurance risk. Insurance: Mathematics and Economics, 51(1), 43-52. http://dx.doi.org/10.1016/j.msmatheco.2012.02.002.

Asmussen, S. (2003). Stead-state properties of GI/G/1. Applied Probability and Queues - Stochastic Modelling and Applied Probability, vol. 51, 266-301.

Bradley, B. O., \& Taqqu, M. S. (2003). Financial risk and heavy tails. Handbook of Heavy Tailed Distributions in Finance, Chapter 2, vol. 1, 35-103. http://dx.doi.org/10.1016/ B978-044450896-6.50004-2.

Cont, R. (2001). Empirical properties of asset returns: stylized facts and statistical issues. Quantitative Finance, vol. 1, 223-236.

Day, M., \& Diamond, M. (2017), GARCH model, heavy tails and the Chinese stock market returns, Working paper.

Glasserman,P., Heidelberger,P., \& Shahabuddin,P.(2002). PortfolioValue-at-Risk with heavytailed risk factors. Mathematical Finance, 12(3), 239-269. http://dx.doi.org/10.1111 /1467-9965.00141.

Guo, Zi-Yi (2017a). Heavy-tailed distribution and risk management of equity market tail events. Journal of Risk \& Control, vol. 4, 31-41. http://dx.doi.org/10.2139/ssrn.301379.

Guo, Zi-Yi (2017b), A Stochastic Factor Model for Risk Management of Commodity Derivatives, Proceedings of the 7th Economic and Finance Conference, 26-42.

Guo, Zi-Yi (2017c), Models with Short-Term Variations and Long-Term Dynamics in Risk Management of Commodity Derivatives, mimeo.

Guo, Zi-Yi (2017d). How Information Is Transmitted across the Nations? An Empirical Investigation of the US and Chinese Commodity Markets. Global Journal of Management and Business Research, 17(2), 1-11.

Hansen, B. (1994). Autoregressive conditional density estimation. International Economic Review, vol. 35, 705-730. http://dx.doi.org/10.2307/2527081.

Huber-Carol, C., Balakrishnan, N., Nikulin, M., \& Mesbah, M. (2002). Goodness-of-Fit Tests and Model Validity, Springer.

Ibragimov, R. (2009). Heavy-tailed densities. The New Palgrave Dictionary of Economics Online.

IMF (2014). Canada financial sector stability assessment. International Monetary Fund Country Report No. 14/29.

Karolyi, G. A. (1995). A multivariate GARCH model of international transmissions of stock returns and volatility: the case of the United States and Canada. Journal of Business \& Economic Statistics, 13(1), 11-25. http://dx.doi.org/10.2307/1392517.

Maree, A. (2017), Regulatory requirements, risk management and the equity market in New Zealand, Working paper.

Maree, A., Carr, G., \& Howard, J. (2017). A new class of heavy-tailed distribution in GARCH models for the silver returns, Journal of Progressive Research in Social Sciences, 5(2), 364-368. 
Mittnik, S., \& Paolella, M. S. (2003). Prediction of financial downside-risk with heavytailed conditional distributions. Handbook of Heavy Tailed Distributions in Finance, Chapter 9, vol. 1, 385-404.

Park, T. H., \& Switzer, L. N. (1995). Bivariate GARCH estimation of the optimal hedge ratios for stock index futures: A note. The Journal of Futures Markets, vol. 15, 61-67. http://dx.doi.org/10.1002/fut.3990150106.

Prause, K. (1999). The generalized hyperbolic model: estimation, financial derivatives, and risk measures. Ph.D. Dissertation.

Ramchand, L., \& Susmel, R. (1998). Volatility and cross correlation across major stock markets. Journal of Empirical Finance, 5(4), 397-416. http://dx.doi.org/10.1016/ S0927-5398(98)0003-6.

Sadorsky, P. (1999). Oil price shocks and stock market activity. Energy Economics, 21(5), 449-469. http://dx.doi.org/10.1016/S0140-9883(99)00020-1.

Taeger, D. \& Kuhnt, S. (2014). Goodness-of-fit tests. Statistical Hypothesis Testing with SAS and R, Wiley Online Library.

Theodossiou, P., \& Unro, L. (1993). Mean and volatility spillovers across major national stock markets: further empirical evidence. The Journal of Financial Research, 16(4), 337-350. http://dx.doi.org/10.1111/j.1475-6803.1993.tb00152.x. 
